\title{
Modulation of gut microbiota by antibiotics improves insulin signalling in high-fat fed mice
}

\author{
B. M. Carvalho • D. Guadagnini • D. M. L. Tsukumo • \\ A. A. Schenka • P. Latuf-Filho • J. Vassallo • J. C. Dias • \\ L. T. Kubota • J. B. C. Carvalheira • M. J. A. Saad
}

Received: 23 February 2012 / Accepted: 18 June 2012 /Published online: 25 July 2012

(C) Springer-Verlag 2012

\begin{abstract}
Aims/hypothesis A high-fat dietary intake induces obesity and subclinical inflammation, which play important roles in insulin resistance. Recent studies have suggested that increased concentrations of circulating lipopolysaccharide (LPS), promoted by changes in intestinal permeability, may have a pivotal role in insulin resistance. Thus, we investigated the effect of gut microbiota modulation on insulin resistance and macrophage infiltration.

Methods Swiss mice were submitted to a high-fat diet with antibiotics or pair-feeding for 8 weeks. Metagenome analyses were performed on DNA samples from mouse faeces. Blood was collected to determine levels of glucose, insulin, LPS, cytokines and acetate. Liver, muscle and adipose tissue proteins were analysed by western blotting. In addition, liver and adipose tissue were analysed, blinded, using histology and immunohistochemistry.

Results Antibiotic treatment greatly modified the gut microbiota, reducing levels of Bacteroidetes and Firmicutes, overall bacterial count and circulating LPS levels. This modulation reduced levels of fasting glucose, insulin, TNF- $\alpha$ and IL-6;
\end{abstract}

Electronic supplementary material The online version of this article (doi:10.1007/s00125-012-2648-4) contains peer-reviewed but unedited supplementary material, which is available to authorised users.

B. M. Carvalho · D. Guadagnini • D. M. L. Tsukumo •

A. A. Schenka $\cdot$ P. Latuf-Filho $\cdot$ J. Vassallo J. B. C. Carvalheira

M. J. A. Saad $(\bowtie)$

Internal Medicine Department-Faculty of Medical Sciences,

State University of Campinas,

Rua Tessália Vieira de Camargo,

126, Cidade Universitária Zeferino Vaz,

Campinas, São Paulo 13083-887, Brazil

e-mail:msaad@fcm.unicamp.br

J. C. Dias $\cdot$ L. T. Kubota

Chemistry Institute, State University of Campinas,

Campinas, São Paulo, Brazil reduced activation of toll-like receptor 4 (TLR4), c-Jun $\mathrm{N}$-terminal kinase (JNK), inhibitor of $\mathrm{k}$ light polypeptide gene enhancer in $B$ cells, kinase $\beta$ (IKK $\beta$ ) and phosphorylated IRS-1 Ser307; and consequently improved glucose tolerance and insulin tolerance and action in metabolically active tissues. In addition, there was an increase in portal levels of circulating acetate, which probably contributed to an increase in 5'-AMP-activated protein kinase (AMPK) phosphorylation in mice. We observed a striking reduction in crown-like structures (CLS) and $\mathrm{F} 4 / 80^{+}$macrophage cells in the adipose tissue of antibiotic-treated mice.

Conclusions/interpretation These results suggest that modulation of gut microbiota in obesity can improve insulin signalling and glucose tolerance by reducing circulating LPS levels and inflammatory signalling. Modulation also appears to increase levels of circulating acetate, which activates AMPK and finally leads to reduced macrophage infiltration.

Keywords Gut microbiota · High-fat diet · Inflammation • Insulin resistance $\cdot$ Lipopolysaccharide

$\begin{array}{ll}\text { Abbreviations } \\ \text { ACC } & \text { Acetyl-CoA carboxylase } \\ \text { Akt } & \text { Thymoma viral proto-oncogene 1 } \\ \text { AMPK } & \text { 5'-AMP-activated protein kinase } \\ \text { BLASTX } & \text { Basic local alignment search tool X } \\ \text { CFU } & \text { Colony-forming units } \\ \text { CLS } & \text { Crown-like structure } \\ \text { F4/80 } & \text { Epidermal-growth-factor-like module- } \\ & \text { containing mucin-like hormone receptor-like 1 } \\ \text { GIR } & \text { Glucose infusion rate } \\ \text { GLP-1 } & \text { Glucagon-like peptide-1 } \\ \text { HFD } & \text { High-fat diet } \\ \text { IKB } \alpha & \text { Nuclear factor of } \kappa \text { light polypeptide gene } \\ \text { IKK } \beta & \text { enhancer in B cells inhibitor, } \alpha \\ & \text { Inhibitor of nuclear factor } \kappa-B \text { kinase subunit } \beta\end{array}$




$\begin{array}{ll}\text { IR } & \text { Insulin receptor } \\ \text { JNK } & \text { c-Jun N-terminal kinase } \\ \text { LPS } & \begin{array}{l}\text { Lipopolysaccharide } \\ \text { Myeloid differentiation primary response } \\ \text { Mene } 88\end{array} \\ & \text { Non-alcoholic steatohepatitis } \\ \text { NASH } & \text { Peptide YY } \\ \text { PYY } & \text { Short-chain fatty acid } \\ \text { SCFA } & \text { Toll-like receptor } 4 \\ \text { TLR4 } & \end{array}$

A combination of environmental factors, including high-fat, high-carbohydrate diets and sedentary lifestyles, have led to dramatic increases in the incidence of obesity and its associated disorders worldwide [1]. The chronic low-grade inflammatory status present in obesity and type 2 diabetes is characterised by increased levels of pro-inflammatory cytokines, acute-phase reactants and other stress-induced molecules, and activation of a network of inflammatory signalling pathways [2].

Recent evidence suggests that gut microbiota are important for the control of body weight and energy homeostasis. Several mechanisms have been proposed for this interaction, but they are not well defined. Increased energy harvesting from gut flora was suggested on the basis of studies in which gut microbiota from lean mice were transplanted to germ-free animals, which then exhibited rapid weight gain, dramatic increases in total and epididymal fat content, and a reduction in lean body mass, even with a lower food intake. These animals went on to develop insulin resistance [3]. Focusing on gut microbiota profiles, Turnbaugh and colleagues [4] showed different bacteria prevalence in lean vs obese mice, as well as in humans, by metagenomic analysis, relying on the presence of increased energy harvesting from obesity-associated gut microbiota. Another proposed mechanism is suppression of intestinal fasting-induced adipose factor (FIAF), which is an adipose tissue lipoprotein lipase (LPL) inhibitor that limits adipocyte uptake of fatty acids and triacylglycerol accumulation, and modulates peroxisomal proliferator-activated receptor coactivator $1 \alpha$ (PGC$1 \alpha)$ which, in turn, activates key enzymes involved in mitochondrial fatty acid oxidation. 5'-AMP-activated protein kinase (AMPK) is persistently activated in germfree mice, and is reduced when microbiota are transplanted to these animals, consequently reducing mitochondrial fatty acid oxidation [3]. Most of these mechanisms may contribute to the ways in which gut microbiota can modulate fat storage and energy homeostasis [4-7]. However, the connections between gut microbiota and insulin sensitivity and signalling are incompletely understood.

In theory, gut microbiota might regulate insulin sensitivity by multiple mechanisms but, clearly, lipopolysaccharides (LPSs) and short-chain fatty acids (SCFAs) from gut microbiota, signalling through toll-like receptor 4 (TLR4) and AMPK, respectively, in host tissues, are potentially important candidates for mediating this modulation. However, the correlation between changes in gut microbiota and the influence of these signalling pathways in insulin sensitivity has not yet been investigated.

It has been established that a high-fat diet (HFD) can modulate gut microbiota in mice, altering the percentages of particular species of bacteria. In addition, antibiotics can downregulate gut microbiota dramatically, reducing the number of colonies and also modulating some bacterial species. The aim of the present study was to use these approaches to gut microbiota modulation - HFD and/or antibiotics - to investigate correlations between changes in microbiota and insulin sensitivity and signalling, and, at the same time, the possible mechanism that accounts for these effects.

\section{Methods}

Materials Antibodies were purchased from Santa Cruz Biotechnology (Santa Cruz, CA, USA), except for anti-nuclear factor of $k$ light polypeptide gene enhancer in $\mathrm{B}$ cells inhibitor, $\alpha(\mathrm{I} \kappa \mathrm{B} \alpha)$, anti-phosphorylated AMPK, antiAMPK, anti-acetyl-CoA carboxylase (ACC), antiphosphorylated ACC and anti-TLR4 were purchased from Cell Signaling Technology (Beverly, MA, USA). Human recombinant insulin (Humulin) was purchased from Eli Lilly (Indianapolis, IN, USA). Routine reagents were purchased from Sigma-Aldrich (Saint Louis, MO, USA), unless otherwise specified.

Animals Six-week-old male Swiss mice were obtained from the UNICAMP Central Animal Breeding Centre (Campinas, São Paulo, Brazil). Animals ( $n=6$ per group) were housed in individual cages with free access to water and rodent chow under a $12 \mathrm{~h} \mathrm{light/dark} \mathrm{cycle.} \mathrm{Diet-induced} \mathrm{obesity} \mathrm{was}$ promoted by feeding mice with an HFD for 12 weeks. The HFD consisted of 55\% of energy derived from fat, $29 \%$ from carbohydrates and 16\% from protein. All procedures were approved by the Ethics Committee of the State University of Campinas.

Antibiotic treatment Mice fed the HFD received antibiotic treatment (ampicillin, neomycin and metronidazole, each at $1 \mathrm{~g} / \mathrm{l})$ in place of drinking water for 8 weeks.

Pair-fed mice Animals fed the HFD were pair-fed to prevent weight differences between groups of mice.

Metagenome profile Faecal samples were frozen in liquid nitrogen and stored at $-80^{\circ} \mathrm{C}$ until use. At this time, DNA 
was extracted using the QIAamp DNA Stool Mini Kit (Qiagen, Hilden, Germany) and quantified. Libraries were synthesised from 500 ng total DNA following Rapid Library Preparation Kit instructions (Roche Applied Science, Mannheim, Germany). These libraries were analysed in a bioanalyser using the High Sensitive DNA Kit (Agilent Technologies, Santa Clara, CA, USA), and equimolar pools were made, titrated and submitted to large-volume PCR following the manufacturers' instructions (Roche Applied Science, Mannheim, Germany). Samples were then sequenced in GS FLX Titanium using the GS FLX Titanium PicoTiterPlate Kit combined with the GS FLX Titanium Sequencing Kit XLR70 (Roche Applied Science, Mannheim, Germany). The readouts obtained from the sequencing were analysed by bioinformatics using basic local alignment search tool X (BLASTX), observed with METAREP software, and compared according to phylum prevalence among groups.

Culture-based microbial analysis of caecal contents Total aerobic and anaerobic bacteria were enumerated in selective media and incubation conditions according to Schumann et al [8]. Briefly, caecal samples were diluted in Ringer medium, and total aerobic and anaerobic bacteria were investigated by plating onto trypcase soy agar $+5 \%$ sheep blood (TSS) medium (Biomerieux, Lyon, France) for $24-48 \mathrm{~h}$ at $37^{\circ} \mathrm{C}$ under aerobic and anaerobic conditions. Bacterial numbers were expressed as colony forming units (CFU)/g caecal content [9].

SCFA determination Blood was withdrawn and centrifuged $(1,000 \mathrm{~g}$ for $15 \mathrm{~min})$. Serum was deproteinised with methanol (1:5 dilution), and centrifuged at 50,000 $\mathrm{g}$ for $10 \mathrm{~min}$ at $4{ }^{\circ} \mathrm{C}$. The supernatant fraction was separated chromatographically using an Acquity ultra performance liquid chromatography (UPLC), a BEH amide column $(2.1 \times 100 \mathrm{~mm}$, $1.7 \mu \mathrm{m})$ and injected in a Quattro Micro API mass spectrometer (Waters, Milford, MA, USA) for measurement of acetate concentration.

Insulin tolerance test Mice were fasted for $8 \mathrm{~h}$ and subjected to a $30 \mathrm{~min}$ insulin tolerance test $(1.5 \mathrm{U} / \mathrm{kg})$; the glucose disappearance rate was calculated as previously described [10].

Intraperitoneal glucose tolerance test After fasting the mice for $8 \mathrm{~h}$, a glucose tolerance test was performed. Glucose $(1 \mathrm{~g} / \mathrm{kg})$ was injected intraperitoneally and blood samples were collected from the tail vein at different time points up to 120 min to determine blood glucose levels.

Assays ELISA kits were used to determine insulin (Millipore, St Charles, MO, USA), TNF- $\alpha$ and IL-6 (Thermo Fisher Scientific, Rockford, IL, USA).
Hyperinsulinaemic-euglycaemic clamp After an overnight fast, mice were anaesthetised and catheterised. A $120 \mathrm{~min}$ hyperinsulinaemic-euglycaemic clamp was carried out with a prime continuous insulin infusion $\left(30 \mathrm{mU} \mathrm{kg}^{-1} \mathrm{~min}^{-1}\right)$. Blood glucose was measured at 5 min intervals and glucose $(5 \% \mathrm{wt} / \mathrm{vol}$.) was infused at a variable rate to maintain blood glucose at fasting levels.

LPS determination Sterile serum samples, obtained from portal vein blood, were diluted to $20 \%$ (vol./vol.) with endotoxin-free water and then heated to $70^{\circ} \mathrm{C}$ for $10 \mathrm{~min}$ to inactivate serum proteins. LPS was quantified using a commercially available Limulus Amebocyte Assay (Cambrex, Walkersville, MD, USA), according to the manufacturer's protocol.

Sodium acetate administration Sodium acetate was administered to lean mice by oral gavage $(1 \mathrm{~g} / \mathrm{kg}) 30$ and $60 \mathrm{~min}$ before tissue extraction or by venous infusion. For the infusion, lean mice were anaesthetised, a catheter inserted into the jugular vein and the solution containing $6 \mu \mathrm{g}$ of sodium acetate was infused with a syringe pump (KD Scientific, Holliston, MA, USA). After the specified time of the oral gavage and immediately after acetate infusion, blood was withdrawn and liver, muscle and adipose tissue homogenised in specific extraction buffer.

Tissue extraction, immunoprecipitation and protein analysis by immunoblotting Mice fasted for $8 \mathrm{~h}$ were anaesthetised, the abdominal cavity was opened, the vena cava exposed and $\mathrm{NaCl}\left(0.9 \% \mathrm{wt} / \mathrm{vol}\right.$.) solution or insulin $\left(10^{-6} \mathrm{~mol} / \mathrm{l}\right)$ was injected. Liver, muscle and adipose tissue were removed, minced coarsely and homogenised in specific extraction buffer. Tissue extracts were incubated overnight with myeloid differentiation primary response gene 88 (MyD88) and IRS-1 antibody and immunoprecipitated using protein A Sepharose 6MB (GE Healthcare, Uppsala, Sweden). The immunoprecipitates and whole-tissue extracts were subjected to SDS-PAGE and immunoblotted with specific antibodies. Immunoreactive bands were detected by chemiluminescence (Supersignal West Pico Chemiluminescent Substrate, Thermo Fisher Scientific).

Liver and adipose tissue histology Liver and epididymal white adipose tissue were dissected and fixed by immersion in $4 \%(\mathrm{wt} / \mathrm{vol}$.) paraformaldehyde in phosphate buffer, $\mathrm{pH} 7.4$ for $24 \mathrm{~h}$, dehydrated, cleared and then embedded in paraffin. Sections $(5 \mu \mathrm{m})$ were obtained and stained by haematoxylin and eosin to assess morphology. Tissue analyses were performed blinded by an expert pathologist (AAS). Adipose tissue was evaluated by crown-like structure (CLS) density (average CLS number within ten high-power fields 
per animal) and mean adipocyte surface area, using Imagelab Analysis software, as previously described [11].

Liver and adipose tissue immunohistochemistry Tissue sections, $5 \mu \mathrm{m}$, were mounted on silanised glass slides, processed and incubated with epidermal-growth-factor-like modulecontaining mucin-like hormone receptor-like 1 (F4/80) primary antibody, as previously described [12]. Antibody staining was performed using an IHC-peroxidase kit (Advance HRP, Dako CytoMation, Carpinteria, CA, USA) according to the manufacturer's instructions. Three different high-power fields from three different sections were evaluated blinded by an expert pathologist (AAS). The total number of nuclei of F4/ 80 -expressing cells was counted for each field and the area occupied by these cells was measured.

Statistical analysis Results are expressed as mean \pm SEM. Blot results are presented as direct comparisons of bands in autoradiographs quantified by optical densitometry (UNSCAN-IT gel; Silk Scientific, Orem, UT, USA). Statistical analyses were performed using a two-way ANOVA with the Bonferroni test for post hoc comparisons and Student's $t$ test where necessary. A $p$ value $<0.05$ was considered statistically significant.

\section{Results}

Effect of antibiotic treatment on gut microbiota Based on BLASTX analysis of metagenomic sequencing from faeces of all groups, we observed a profile change between rodents fed chow and the HFD. The prevalence of Bacteroidetes increased in the gut of high-fat-fed mice compared with control mice. In contrast, the presence of Firmicutes was significantly reduced in animals that received the HFD. In addition, the latter group showed a marked increase in the prevalence of Verrucomicrobia bacteria compared with control mice. Metagenomic studies revealed that after treatment with broad-spectrum antibiotics, the gut microbiota profile was profoundly altered, showing almost no Bacteroidetes or Verrucomicrobia and a low prevalence of Firmicutes. These modifications were accompanied by a huge increase in the prevalence of Proteobacteria (Fig. 1a-c). Besides this modulation of gut microbiota, bacterial quantification in faeces from antibiotic-treated mice showed striking overall reductions compared with untreated animals (Fig. 1d).

Effect of antibiotic treatment on metabolic variables of mice fed an HFD Antibiotic-treated mice receiving an HFD had a reduced food intake compared with animals that did not receive antibiotics, gaining significantly less weight during the 8 weeks of the experiment (data not shown). In order to avoid this bias and not mask the treatment effect on insulin
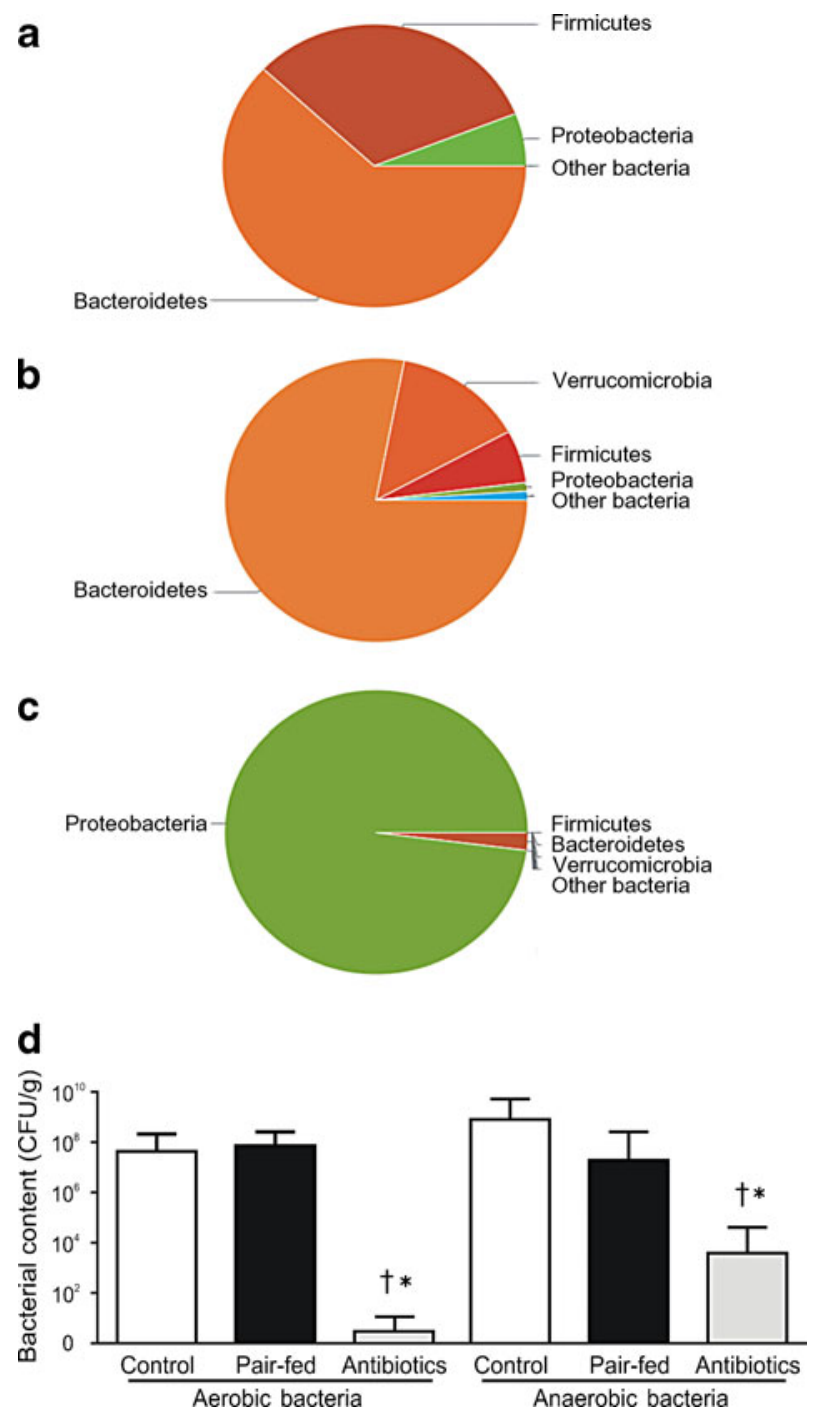

Fig. 1 Metagenomic analysis of gut microbiota prevalence in control (a), pair-fed (b) and antibiotic-treated mice (c). Bacterial counts were taken from control, pair-fed and antibiotic-treated mouse faeces (d). Phylum charts were extracted using METAREP software based on BLASTX analysis of DNA pyrosequencing from mouse faeces. Each bar represents the mean \pm SEM from six mice per group. ${ }^{*} p<0.05$ compared with pair-fed mice; ${ }^{\dagger} p<0.05$ compared with control mice

signalling, we submitted the non-treated animals to pairfeeding on an HFD. The different groups of mice did not show significant weight differences (Fig. 2a). Even under these experimental conditions, the pair-fed group showed increased fasting blood glucose and serum insulin levels compared with control and antibiotic-treated animals (Fig. 2b,c). In mice receiving an HFD and treated with antibiotics, there was an improvement in insulin tolerance, determined by the insulin tolerance test, and in glucose tolerance measured by the intraperitoneal glucose tolerance test (Fig. 2d,e). Mice with diet-induced obesity showed a striking reduction in the glucose infusion rate (GIR) measured by the hyperinsulinaemic-euglycaemic clamp 
Fig. 2 The effect of antibiotic treatment on mice receiving an HFD. Body weight (a), fasting blood glucose (b), portal serum insulin (c), insulin sensitivity based on KITT (K value for insulin tolerance test, which provides a measure of glucose disappearance rate) (d), and glucose tolerance testing (e) of control, pair-fed and antibiotictreated mice. Hyperinsulinaemic-euglycaemic clamp of chow-fed mice, HFD-fed mice, antibiotic-treated HFD-fed mice and after LPS injection (f). Each bar represents the mean \pm SEM from six mice per group. In (a) and (e), circle, control; square, pair-fed; and triangle, antibiotic. ${ }^{*} p<0.05$ compared with pair-fed mice; ${ }^{\dagger} p<0.05$ compared with mice receiving HFD + antibiotics; ${ }^{\star} p<0.05$ compared with control mice

\section{a}

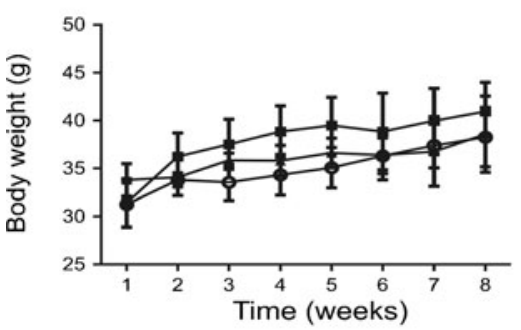

c
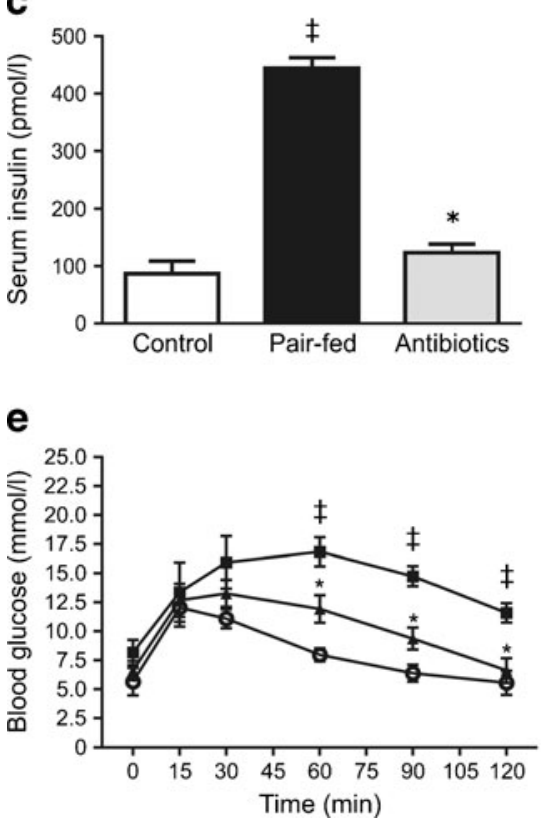

b

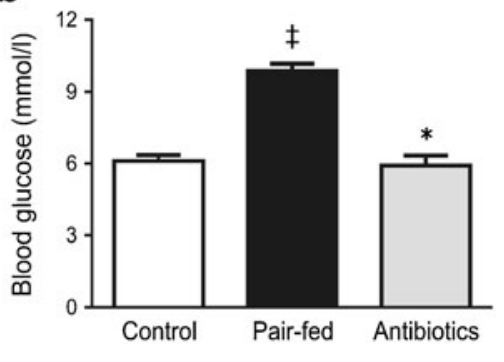

d

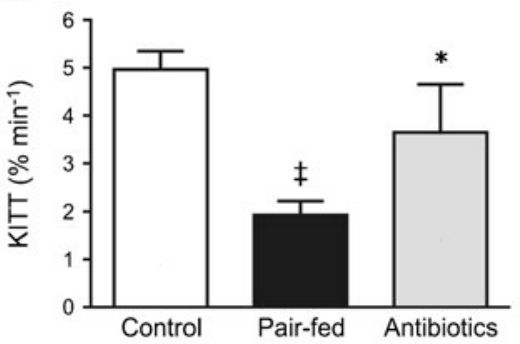

$\mathbf{f}$

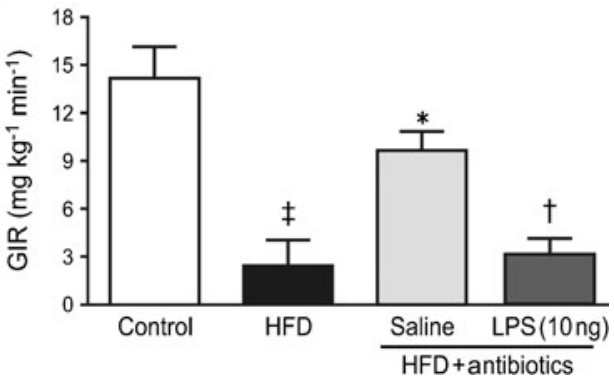

compared with control animals. After antibiotic treatment for 1 week, the insulin sensitivity was partially restored. However, after an LPS injection (10 ng), the GIR was reduced to the levels of the obese mice, demonstrating an important action of intestinal LPS in obesity-induced insulin resistance (Fig. 2f).

Effect of antibiotic treatment on LPS, proinflammatory cytokines and portal acetate levels Mice fed the HFD under the pair-fed regimen showed increased levels of LPS in their portal circulation $(p<0.00001)$ compared with the control group; this was prevented by antibiotic treatment (Fig. 3a). The serum TNF- $\alpha$ and IL- 6 levels showed increases in untreated mice given the HFD compared with the control group, and antibiotic treatment blocked these increases in cytokine level (Fig. 3b,c). In addition, concentrations of serum acetate were significantly reduced in mice fed the HFD compared with mice receiving standard chow; after antibiotic treatment, levels remained close to those in control animals (Fig. 3d).

Effect of antibiotic treatment on the TLR4 signalling pathway in HFD-fed mice The increased circulating concentration of
LPS absorbed from the gut upregulated the TLR4 signalling pathway in liver, muscle and adipose tissue in mice
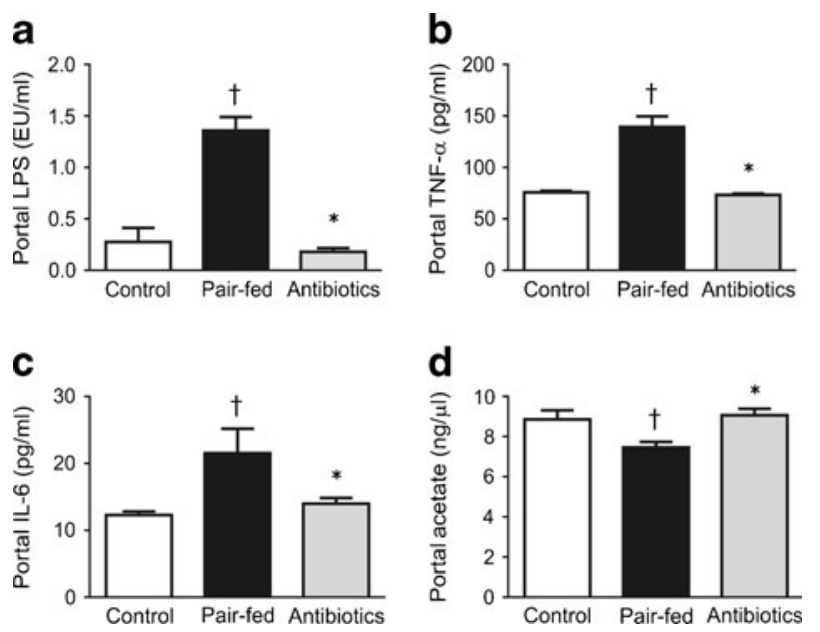

Fig. 3 The effect of antibiotics on portal vein serum levels of LPS (a), TNF- $\alpha$ (b), IL-6 (c) and acetate (d) from control, pair-fed and antibiotic-treated mice. Each bar represents the mean \pm SEM from six mice per group. ${ }^{*} p<0.05$ compared with pair-fed mice; ${ }^{\dagger} p<0.05$ compared with control mice 
subjected to a pair-feeding regimen. This event resulted in increased c-Jun N-terminal kinase (JNK) activation and I $\mathrm{KB} \alpha$ degradation, consequently enhancing IRS-1 Ser307 phosphorylation, which in turn induced insulin resistance. Modulation of gut microbiota by antibiotic treatment reduced TLR4 activation by reducing levels of circulating LPS, consequently decreasing JNK activation, IKB $\alpha$ degradation and IRS-1 Ser307 phosphorylation (Fig. 4a-1).

Effect of antibiotic treatment on the insulin signalling pathway and AMPK Pair-fed mice showed inflammationinduced impairment of the insulin signalling pathway after stimulation in the liver, muscle and adipose tissue, represented by reductions in insulin-induced insulin receptor (IR), IRS-1 and Akt phosphorylation. After antibiotic treatment, insulin-induced phosphorylation of these proteins increased, followed by reductions in LPS concentration and observed inflammation, which promoted amelioration of insulin signalling (Fig. 5a-i). In mice fed an HFD, there were decreases in AMPK and ACC phosphorylation, which were increased after antibiotic treatment (Fig. 6a-f).

Effect of acetate on the AMPK and ACC phosphorylation In order to elucidate whether acetate induces AMPK and ACC
Fig. 4 The effect of antibiotics on TLR4 activation $(\mathbf{a}, \mathbf{e}, \mathbf{i})$ and its downstream effectors JNK $(\mathbf{b}, \mathbf{f}, \mathbf{j})$ and $\mathrm{I} \kappa \mathrm{B} \alpha$ degradation $(\mathbf{c}, \mathbf{g , k}, \mathbf{k})$, leading to ser307 phosphorylation of IRS-1 $(\mathbf{d}, \mathbf{h}, \mathbf{l})$ in liver $(\mathbf{a}-\mathbf{d})$, muscle (e-h) and adipose tissue (i-l). Each bar represents the mean \pm SEM of densitometric quantification of blots from three different experiments. ${ }^{*} p<0.05$ compared with pair-fed mice; ${ }^{\dagger} p<0.05$ compared with control mice. IB, immunoblot; IP, immunoprecipitation
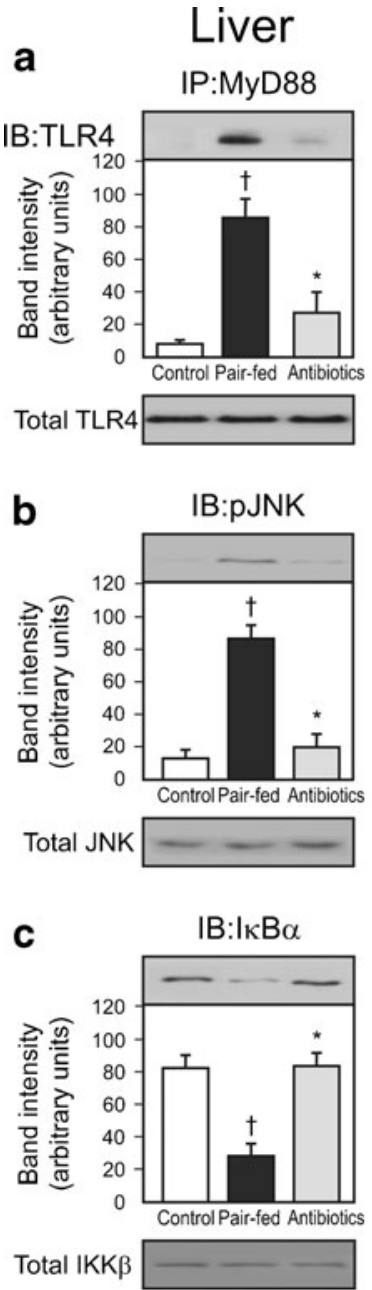

d IB:pIRS-1 (Ser307)

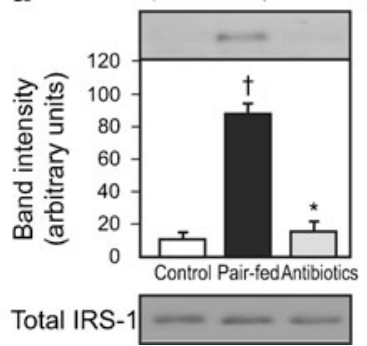

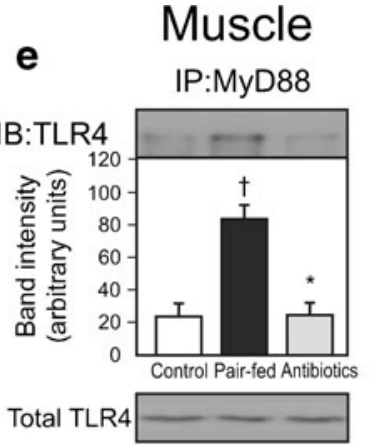

f

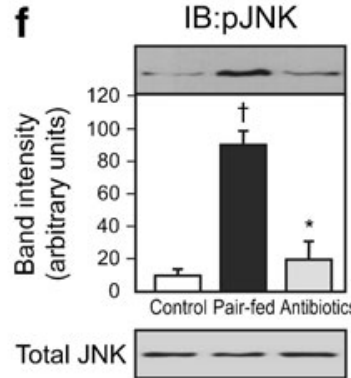

g

$\mathrm{IB}: \mathrm{I} \kappa \mathrm{B} \alpha$

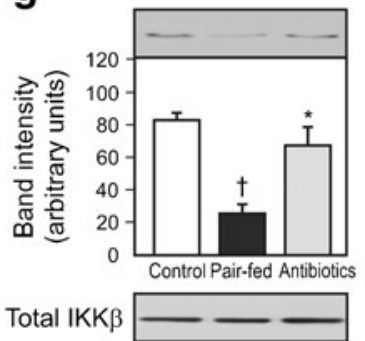

h IB:pIRS-1 (Ser307)

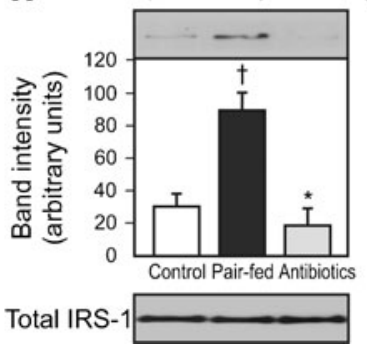

Adipose

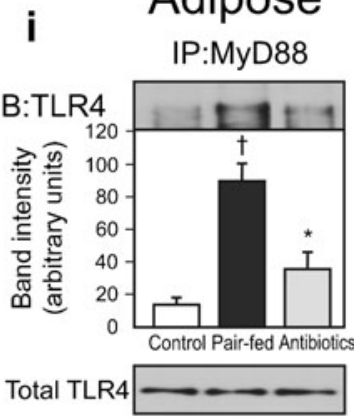

j

IB:pJNK

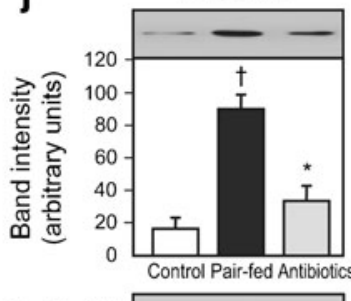

Total JNK

k

IB: $1 \kappa \mathrm{B} \alpha$

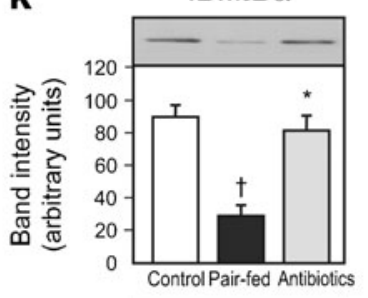

Total IKK $\beta$
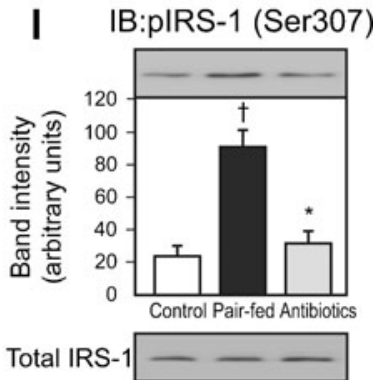
Fig. 5 The effect of antibiotics on the insulin signalling pathway, IR (a,d,g), IRS-1 (b,e, h) and Akt $(\mathbf{c}, \mathbf{f}, \mathbf{i})$ in liver $(\mathbf{a}-\mathbf{c})$, muscle $(\mathbf{d}-\mathbf{f})$ and adipose tissue $(\mathbf{g}-\mathbf{i})$ of mice under the pair-feeding regimen and receiving antibiotic treatment. Each bar represents the mean \pm SEM of densitometric quantification of blots from three different experiments. ${ }^{* *} p<0.01$ compared with pair-fed mice stimulated with insulin
Liver
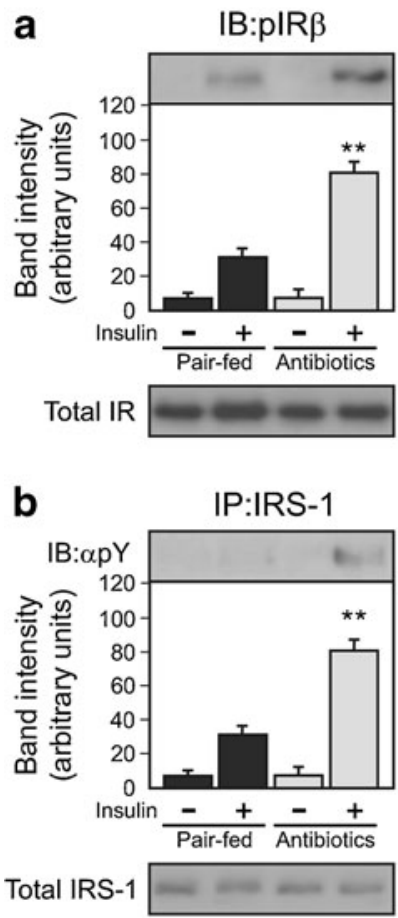

C

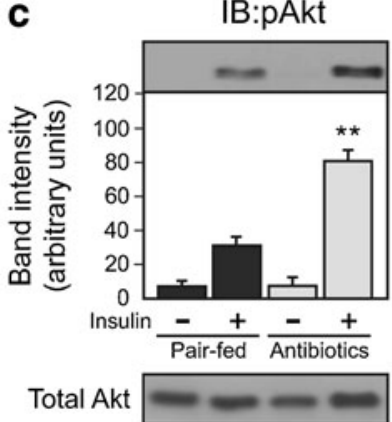

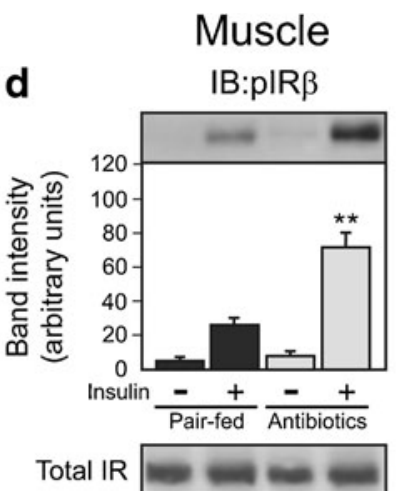

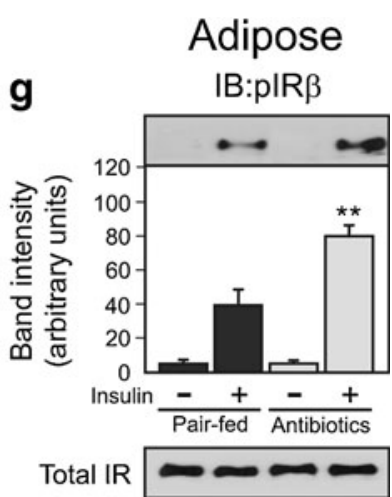

e

IP:IRS-1

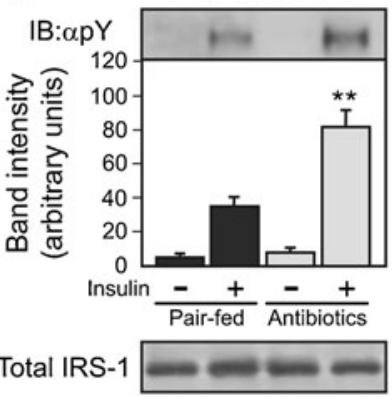

h

IP:IRS-1

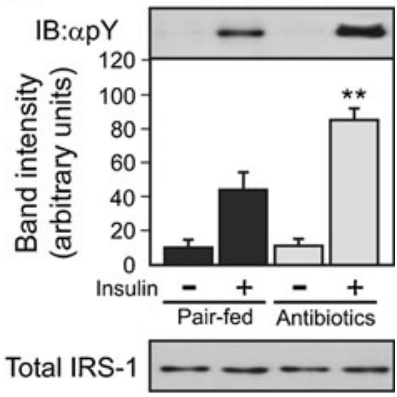

f

IB:pAkt

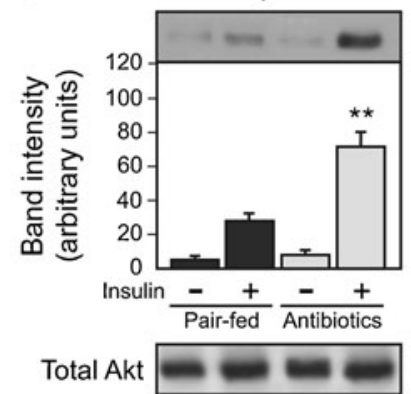

i

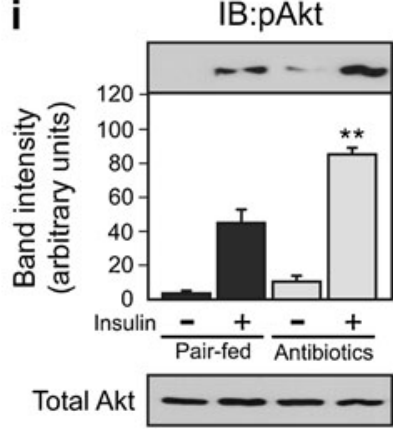

phosphorylation, we treated lean mice with an oral sodium acetate bolus and venous infusion and evaluated the phosphorylation states of these proteins. We observed that 60 min after sodium acetate administration, AMPK and ACC were phosphorylated in the liver, muscle and adipose tissue, and similar results were obtained in sodium acetateinfused mice (Fig. 6g-1).

Effect of antibiotic treatment on histology and macrophage infiltration in liver Microscopic assessment of liver samples revealed that the steatosis induced by the HFD in the antibiotic-treated group was slightly reduced in comparison with the pair-fed group. The number of Küpffer cells increased twofold in pair-fed mice, as detected by $\mathrm{F} 4 / 80^{+}$ staining immunohistochemistry. This macrophage infiltration was inhibited by antibiotic treatment, remaining at a basal count of Küpffer cells (Fig. 7a-e).
Effect of antibiotic treatment on histology and macrophage infiltration in adipose tissue Evaluation of adipose tissue cells revealed no differences in adipocyte size between pair-fed and antibiotic-treated groups, probably due to the absence of body weight differences (data not shown). However, a striking increase in CLSs was found in the adipose tissue of pair-fed mice when compared with the treatment group. Striking increases in the number of infiltrated macrophages, determined by $\mathrm{F} 4 / 80^{+}$ staining, were also observed in the adipose tissue of pair-fed mice, but such cells were virtually eliminated from the vicinity of adipocytes by the antibiotic treatment (Fig. 8a-f).

\section{Discussion}

Here we show that an HFD changed gut microbiota in mice, inducing an increase in the percentage of Bacteroidetes that 
Fig. 6 The effect of antibiotics on AMPK and ACC phosphorylation in the liver $(\mathbf{a}, \mathbf{d})$, muscle $(\mathbf{b}, \mathbf{e})$ and adipose tissue $(\mathbf{c}, \mathbf{f})$. The effect of sodium acetate administration 30 and 60 min after oral gavage and immediately after venous infusion on AMPK and ACC phosphorylation in the liver $(\mathbf{g}, \mathbf{j})$, muscle $(\mathbf{h}, \mathbf{k})$ and adipose tissue (i,l). Each bar represents the mean \pm SEM of densitometric quantification of blots from three different experiments. ${ }^{*} p<0.05$ compared with pair-fed mice; ${ }^{\dagger} p<0.05$ compared with control mice with acetate at $30 \mathrm{~min}$; ${ }^{\sharp} p<0.05$ compared with control mice. v.o., after oral gavage; i.v., immediately after venous infusion
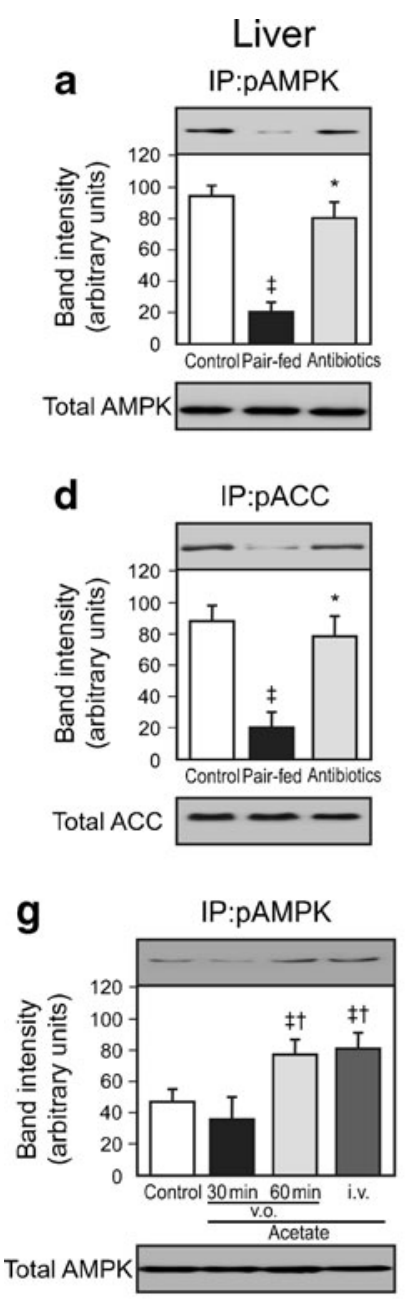

j

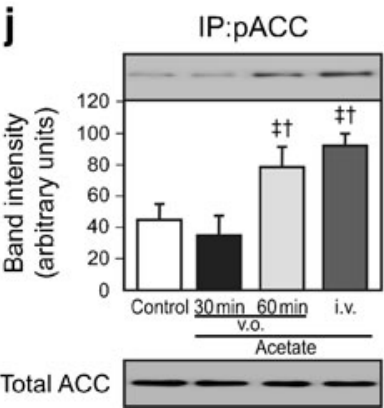

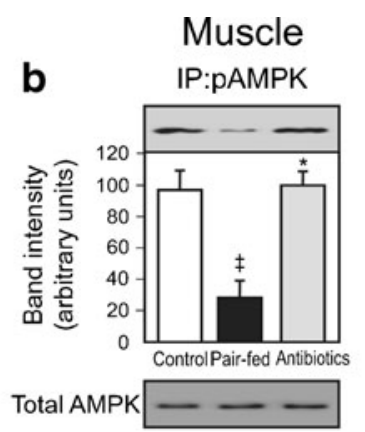

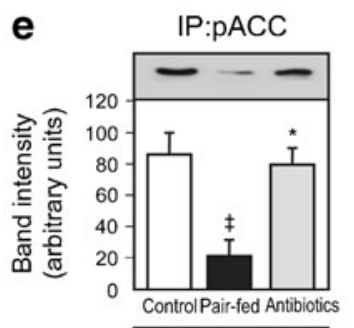

Total ACC

h

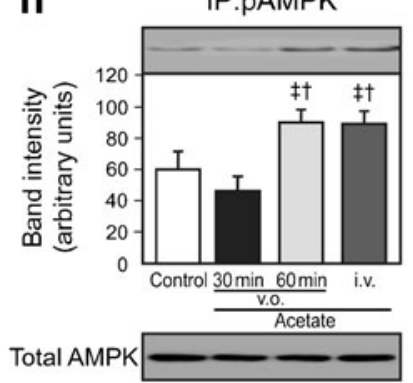

k

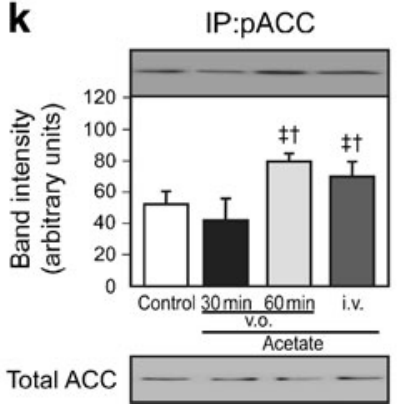

Adipose
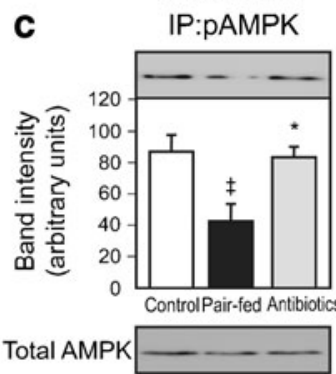

f

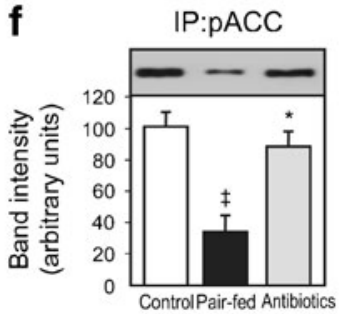

Total ACC

$$
\text { i }
$$

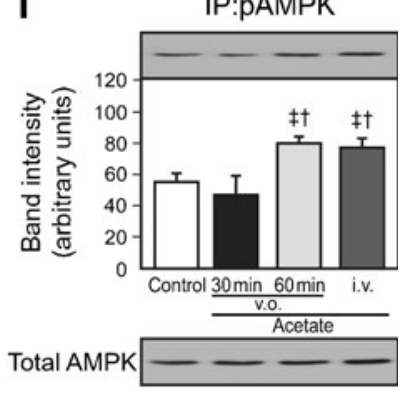

I

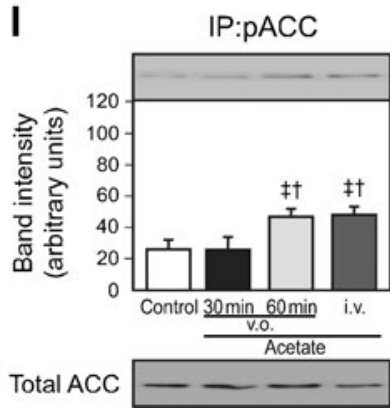

was associated with an increase in circulating LPS levels, a reduction in circulating acetate and a decrease in wholebody insulin tolerance. When these animals were treated with antibiotic as a strategy for intestinal flora modulation, they showed marked reductions in gut bacteria content and circulating LPS, and increases in SCFA levels, which attenuated TLR4 activation. This attenuation, in turn, resulted in improved insulin signalling and inflammatory profiles, and activation of AMPK in hepatic, muscular and adipose tissue. In addition, striking reductions in macrophage infiltration of the liver and fat pad were noted, culminating in prevention of insulin resistance.
Our metagenomic analysis of gut microbiota in HFD-fed mice showed a slight increase in Bacteroidetes prevalence (78.5\% vs $61.8 \%)$ and a significant decrease in Firmicutes (6.1\% vs $31.6 \%)$ compared with the control group. This result was similar to results in previous studies [13-15] that analysed gut microbiota composition in obese and overweight individuals and mice with non-alcoholic steatohepatitis (NASH), but was not in agreement with other previously published data [4, 6]; this is evidence that the gut microbiota profile in obesity is still controversial, and it is possible that differences in the methods used may explain the inconsistencies in the published data. The Verrucomicrobia phylum, 


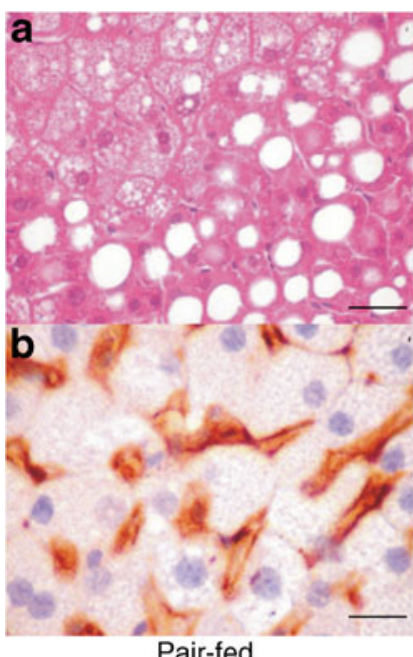

Pair-fed

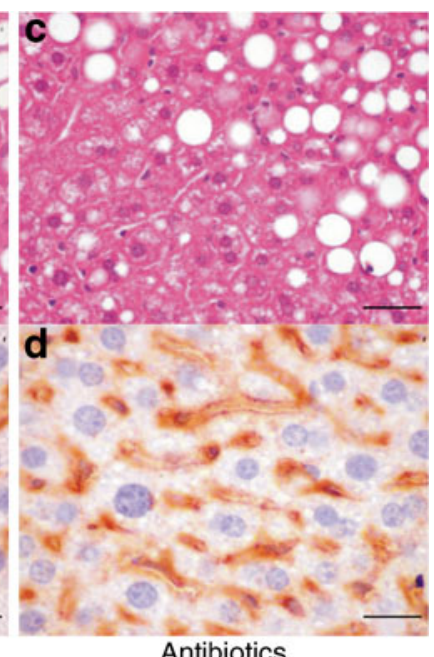

Antibiotics

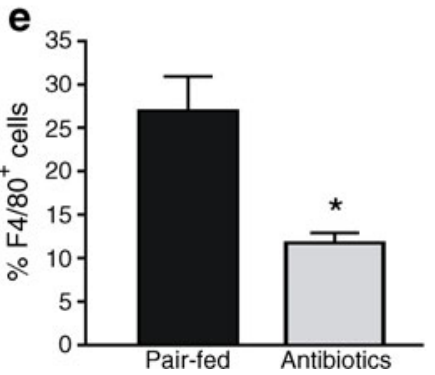

Fig. 7 The effect of antibiotics on liver steatosis (a,c) and macrophage infiltration (b,d), and quantification of $\mathrm{F} 4 / 80^{+}$cells (e) from pair-fed or antibiotic-treated mice. Bars represent the mean \pm SEM from three different experiments. HE staining (a,c) and F4/80 immunostaining (b,d). Magnification $\times 400$; scale bars $50 \mu \mathrm{m}(\mathbf{a}, \mathbf{c})$ and $82.5 \mu \mathrm{m}(\mathbf{b}, \mathbf{d})$. ${ }_{p}^{*}<0.05$ compared with pair-fed mice. Micrographs are representative of six mice per group

which has two Gram-negative sister phyla, Chlamydiae and Lentisphaerae, has not been much explored in the literature, is present in low percentages in control mice, but showed considerable prevalence in animals subjected to an HFD $(0.03 \%$ vs $13.65 \%$ ). Mice subjected to antibiotic treatment showed a huge difference in gut microbiota profiles compared with control and pair-fed groups. Almost all Bacteroidetes and Firmicutes were eradicated from the intestinal lumen, the same fate as for Verrucomicrobia in antibiotic-treated mice, but these changes were accompanied by a striking increase in Proteobacteria, which was almost the sole phylum present (97\%). Even with the high prevalence of Gram-negative bacteria (Proteobacteria), the reduction in circulating levels of LPS is related to the striking reduction in the overall content of bacteria in the intestinal lumen, consequently reducing its uptake, in addition to improving the intestinal barrier function.

Our data showing an increase in circulating portal levels of LPS in mice fed an HFD suggest that gut microbiota are the source of LPS. The data available indicate that translocation of TLR4 agonists, such as LPS, from the gut is an important feature in metabolic diseases such as obesity and
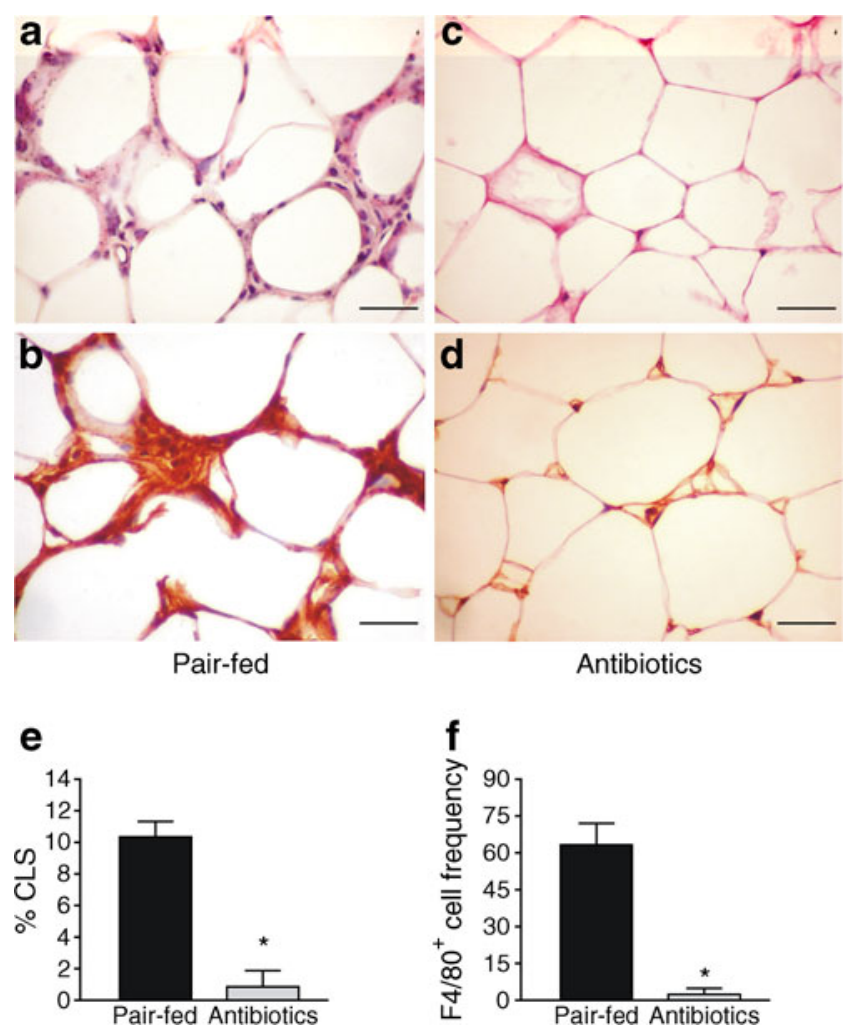

Fig. 8 The effect of antibiotics on the prevalence of CLSs and macrophage infiltration in epididymal adipose tissue (a-e) and quantification of the frequency of $\mathrm{F} 4 / 80^{+}$cells in tissues (f) from pair-fed $(\mathbf{a}, \mathbf{b})$ and antibiotic-treated (c,d) mice. HE staining (a,c) and F4/80 immunostaining $(\mathbf{b}, \mathbf{d})$. Each bar represents the mean \pm SEM from three different experiments. Magnification $\times 400$; scale bar $50 \mu \mathrm{m}(\mathbf{a}-\mathbf{d})$. ${ }^{*} p<0.05$ compared with pair-fed mice

$\mathrm{NASH}$, promoting inflammation and insulin resistance in metabolically active tissues $[15,16]$. In the present study, we showed that the reduction in circulating LPS levels induced by antibiotic treatment in diet-induced obese mice increased insulin sensitivity, and that this was attenuated by a LPS injection, indicating an important role of bacterial LPS in the phenomenon of insulin resistance.

A great deal of data, gained using LPS injection and sepsis models, have shown the importance of LPS in inflammation and the induction of insulin resistance [17-23]. The primary mechanism involving LPS is initiated when it binds TLR4, its receptor, which, in turn, associates with MyD88 and triggers its signalling pathway; this, in turn, activates JNK and inhibitor of nuclear factor $\mathrm{K}$-B kinase subunit $\beta$ (IKK $\beta$ ) through IL-1 receptor-associated kinase (IRAK), leading to serine phosphorylation of IRS-1, nuclear factor $k B(N F-k B)$ activation, transcription of proinflammatory cytokines and insulin resistance [24, 25].

Cani and colleagues [26] showed that antibiotic treatment improved inflammation, oxidative stress and macrophage infiltration markers based on adipose tissue mRNA of rodents fed either regular chow or an HFD, with or without 
antibiotics. They also showed marked augmentation of Zo-1 (also known as Tjp1) mRNA, encoding zonula occludens 1 , an important intestinal barrier protein, which correlated with diminished intestinal permeability, reducing circulating LPS levels in antibiotic-treated mice. All those experiments were performed in HFD-fed animals with differences in body weight, and did not exclude the body weight reduction after antibiotic treatment, an important 'insulin-sensitising' feature, as we did in the present study. Our experiments were conducted using pair-feeding in order to exclude body weight differences, a consequence of reduced food intake induced by the antibiotic treatment, and show increased insulin signalling activation after gut microbiota modulation in antibiotic-treated mice on the HFD. This decrease in food intake after antibiotic treatment is not uniformly observed in other studies $[9,26]$, and the differences may be related to the strain of mice, the type of antibiotic used and the period of treatment. In addition, we cannot exclude the possibility that changes in microbiota induced by antibiotics may have affected gut peptides that regulate food intake, such as peptide YY (PYY) and glucagon-like peptide-1 (GLP-1), as previously described [27].

Our results, similar to previous studies on genetically obese $o b / o b$ mice $[9,28]$, showed an improvement in the whole metabolism of mice under an HFD treated with antibiotics, based on increases in insulin sensitivity and glucose tolerance, and reductions in fasting glucose and insulin. We also showed that the amelioration of metabolism is mainly related to a reduction in circulating LPS levels, with serum levels of TNF- $\alpha$ and IL- 6 remaining the same as in animals fed a standard chow diet. Most of the effects induced by the antibiotic treatment are mediated by modulation of gut microbiota and improvement in intestinal barrier function.

We also report, for the first time, the influence of a reduced LPS concentration after gut microbiota modulation by antibiotic treatment upon downregulation of the TLR4MyD88 association in liver, muscle and adipose tissue, leading to reduced activation of JNK, IKK $\beta$ and serine phosphorylation of IRS-1. An inversely related increase in activation of the insulin signalling pathway was seen in all three tissues [29]. These observations show increased control of inflammation and metabolism mediated by augmented insulin activity in target tissues.

Another important issue regarding the relationship between obesity and gut microbiota is SCFA production, which is based on bacterial fermentation of low- and nondigestible carbohydrates in the diet. Reduction of circulating acetate levels in HFD-fed mice may be related to a reduced dietary carbohydrate:fat ratio, which affects SCFA production [30]. Modifications in the gut microbiota profile promoted by antibiotic therapy induced an increase in the portal levels of circulating acetate. Acetate has the capacity to activate AMPK in liver and muscle [31], increasing fatty acid oxidation [32]. In a corroborative way, we showed that after oral administration and venous infusion of acetate in mice, phosphorylation of AMPK and ACC was increased. There is evidence that organic acids such as butyrate and acetate can contribute to intestinal immune and barrier functions [33] and stimulation of mucin production in the gut [34]. Thus, they could also contribute to the reduced intestinal permeability and lower circulating LPS levels seen in antibiotic-treated mice compared with pair-fed mice.

In the liver, acetate may have a role in AMPK activation, based on the increased levels of SCFA in the portal circulation. However, in other peripheral tissues, such as muscle and adipose tissue, the increase in acetate is probably not the main regulator, as we observed that there is no difference in circulating acetate levels among control, high-fat-fed and antibiotic-treated mice (electronic supplementary material [ESM] Fig. 1a). In this regard, other factors, such as the gut hormone GLP-1, which might be modulated by changes in gut microbiota [35], may also have a role in the activation of AMPK [36].

It seems paradoxical that a decrease in the total bacteria responsible for SCFA production resulted in an increase in portal acetate levels. However, the decrease in anaerobic bacteria, the main acetate producers, was not as dramatic as the decrease in aerobic bacteria (Fig. 1d) and this would help to keep the acetate production at the same level as in mice not receiving antibiotics and the HFD. It is also possible that a reduction in the use of acetate by intestinal cells and/or an increase in intestinal SCFA transport might have a role in this process, but these points deserve further investigation.

Macrophage activity is related to inflammation and the induction of insulin resistance in metabolically active tissues in obesity and type 2 diabetes, enhancing TNF- $\alpha$ and IL- 6 production and activity, mainly in liver and adipose tissue. These are sites preferentially infiltrated by innate immune cells in order to maintain homeostasis and prevent the metabolic dysfunction provoked by high levels of circulating free-fatty acids, glucose and LPS as observed in obese individuals [37, 38].

The reductions in macrophage infiltration in liver and adipose tissue in antibiotic-treated mice, as determined by F4/80 staining, are due to reductions in circulating levels of LPS. Although this molecule can cause inflammation, inducing macrophages to secrete TNF- $\alpha$ and IL-6, thereby promoting insulin resistance, it also induces the production and activity of chemoattractant proteins such as monocyte chemotactic protein 1 (MCP-1), which promotes innate cellular immune responses and translocation in response to stimuli, such as HFD-induced adipose tissue hypertrophy and liver steatosis $[39,40]$. It is important to halt the production of inflammatory molecules, which are promoters of insulin resistance, so that macrophage infiltration is 
prevented, and any macrophages already infiltrating do not receive stimuli to remain infiltrated, as occurs with some drugs or with weight loss [41-44].

Taken together, our data show that an HFD promotes alterations in the gut microbiota profile that favour inflammation and an insulin-resistant status, with intestinal barrier disruption and high circulating LPS levels, in addition to intense macrophage activity. All these obesity-related features were reversed by changes in the gut microbiota profile induced by antibiotic therapy in mice subjected to an HFD. These observations lead us to suggest that strategies that target modification of the gut microbiota profile could bring immense benefits in preventing and attenuating obesityrelated symptoms and metabolic derangement.

Acknowledgements The authors thank L. Janeri and J. Pinheiro (Department of Internal Medicine, UNICAMP, Campinas, São Paulo) and R. Souza (Chemistry Institute, UNICAMP, Campinas, São Paulo) for technical assistance and the Institutos Nacionais de Ciência e Tecnologia (INCT)-Bioanalítica.

Funding This work was supported by grants from Fundação de Amparo à Pesquisa do Estado de São Paulo-FAPESP, Conselho Nacional de Pesquisa - CNPq, and Institutos Nacionais de Ciência e Tecnologia (INCT)-Obesidade e Diabetes.

Contribution statement BMC conceived and designed the experiments, researched and interpreted data, contributed to discussions, and wrote, edited and reviewed the article. DG, DMLT, AAS, PL-F, JV and JCD researched and interpreted data and reviewed the article. LTK interpreted data and reviewed the manuscript. JBCC contributed to discussions and reviewed the article. MJAS designed the experiments, interpreted data, contributed to discussions, and edited and reviewed the article. All authors approved the final version.

Duality of interest The authors declare that there is no duality of interest associated with this manuscript.

\section{References}

1. Kahn SE, Hull RL, Utzschneider KM (2006) Mechanisms linking obesity to insulin resistance and type 2 diabetes. Nature 444:840846

2. Wellen KE, Hotamisligil GS (2005) Inflammation, stress, and diabetes. J Clin Invest 115:1111-1119

3. Backhed F, Ding H, Wang T et al (2004) The gut microbiota as an environmental factor that regulates fat storage. Proc Natl Acad Sci USA 101:15718-15723

4. Turnbaugh PJ, Ley RE, Mahowald MA, Magrini V, Mardis ER, Gordon JI (2006) An obesity-associated gut microbiome with increased capacity for energy harvest. Nature 444:1027-1031

5. Turnbaugh PJ, Backhed F, Fulton L, Gordon JI (2008) Dietinduced obesity is linked to marked but reversible alterations in the mouse distal gut microbiome. Cell Host Microbe 3:213-223

6. Ley RE, Backhed F, Turnbaugh P, Lozupone CA, Knight RD, Gordon JI (2005) Obesity alters gut microbial ecology. Proc Natl Acad Sci USA 102:11070-11075
7. Zhang H, DiBaise JK, Zuccolo A et al (2009) Human gut microbiota in obesity and after gastric bypass. Proc Natl Acad Sci USA 106:2365-2370

8. Schumann A, Nutten S, Donnicola D et al (2005) Neonatal antibiotic treatment alters gastrointestinal tract developmental gene expression and intestinal barrier transcriptome. Physiol Genomics $23: 235-245$

9. Membrez M, Blancher F, Jaquet M et al (2008) Gut microbiota modulation with norfloxacin and ampicillin enhances glucose tolerance in mice. FASEB J 22:2416-2426

10. Carvalho-Filho MA, Ropelle ER, Pauli RJ et al (2009) Aspirin attenuates insulin resistance in muscle of diet-induced obese rats by inhibiting inducible nitric oxide synthase production and Snitrosylation of IRbeta/IRS-1 and Akt. Diabetologia 52:24252434

11. Schenka AA, Machado CM, Grippo MC et al (2005) Immunophenotypic and ultrastructural validation of a new human glioblastoma cell line. Cell Mol Neurobiol 25:929-941

12. Cintra DE, Pauli JR, Araujo EP et al (2008) Interleukin-10 is a protective factor against diet-induced insulin resistance in liver. J Hepatol 48:628-637

13. Schwiertz A, Taras D, Schafer K et al (2010) Microbiota and SCFA in lean and overweight healthy subjects. Obesity 18:190 195

14. Collado MC, Isolauri E, Laitinen K, Salminen S (2008) Distinct composition of gut microbiota during pregnancy in overweight and normal-weight women. Am J Clin Nutr 88:894-899

15. Henao-Mejia J, Elinav E, Jin C et al (2012) Inflammasomemediated dysbiosis regulates progression of NAFLD and obesity. Nature 482:179-185

16. Brun P, Castagliuolo I, Di Leo V et al (2007) Increased intestinal permeability in obese mice: new evidence in the pathogenesis of nonalcoholic steatohepatitis. Am J Physiol Gastrointest Liver Physiol 292:G518-G525

17. Cani PD, Neyrinck AM, Fava F et al (2007) Selective increases of bifidobacteria in gut microflora improve high-fat-diet-induced diabetes in mice through a mechanism associated with endotoxaemia. Diabetologia 50:2374-2383

18. Carvalho-Filho MA, Ueno M, Carvalheira JB, Velloso LA, Saad MJ (2006) Targeted disruption of iNOS prevents LPS-induced S-nitrosation of IRbeta/IRS-1 and Akt and insulin resistance in muscle of mice. Am J Physiol Endocrinol Metab 291:E476-E482

19. Oliveira AG, Carvalho BM, Tobar N et al (2011) Physical exercise reduces circulating lipopolysaccharide and TLR4 activation and improves insulin signaling in tissues of DIO rats. Diabetes 60:784 796

20. Calisto KL, Carvalho Bde M, Ropelle ER et al (2010) Atorvastatin improves survival in septic rats: effect on tissue inflammatory pathway and on insulin signaling. PLoS One 5:e14232

21. Tsukumo DM, Carvalho-Filho MA, Carvalheira JB et al (2007) Loss-of-function mutation in Toll-like receptor 4 prevents dietinduced obesity and insulin resistance. Diabetes 56:1986-1998

22. Shi H, Kokoeva MV, Inouye K, Tzameli I, Yin H, Flier JS (2006) TLR4 links innate immunity and fatty acid-induced insulin resistance. J Clin Invest 116:3015-3025

23. Pilon G, Charbonneau A, White PJ et al (2010) Endotoxin mediated-iNOS induction causes insulin resistance via ONOO induced tyrosine nitration of IRS-1 in skeletal muscle. PLoS One 5:e15912

24. Song MJ, Kim KH, Yoon JM, Kim JB (2006) Activation of Tolllike receptor 4 is associated with insulin resistance in adipocytes. Biochem Biophys Res Commun 346:739-745

25. Nguyen MT, Favelyukis S, Nguyen AK et al (2007) A subpopulation of macrophages infiltrates hypertrophic adipose tissue and is activated by free fatty acids via Toll-like receptors 2 and 4 and JNK-dependent pathways. J Biol Chem 282:35279-35292 
26. Cani PD, Bibiloni R, Knauf C et al (2008) Changes in gut microbiota control metabolic endotoxemia-induced inflammation in high-fat diet-induced obesity and diabetes in mice. Diabetes 57:1470-1481

27. De Silva A, Bloom SR (2012) Gut hormones and appetite control: a focus on PYY and GLP-1 as therapeutic targets in obesity. Gut Liver 6:10-20

28. Cani PD, Possemiers S, van de Wiele T et al (2009) Changes in gut microbiota control inflammation in obese mice through a mechanism involving GLP-2-driven improvement of gut permeability. Gut 58:1091-1103

29. Saad MJ, Araki E, Miralpeix M, Rothenberg PL, White MF, Kahn CR (1992) Regulation of insulin receptor substrate-1 in liver and muscle of animal models of insulin resistance. J Clin Invest 90:1839 1849

30. Okazaki Y, Tomotake H, Tsujimoto K, Sasaki M, Kato N (2011) Consumption of a resistant protein, sericin, elevates fecal immunoglobulin a, mucins, and cecal organic acids in rats fed a high-fat diet. J Nutr 141:1975-1981

31. Sakakibara S, Yamauchi T, Oshima Y, Tsukamoto Y, Kadowaki T (2006) Acetic acid activates hepatic AMPK and reduces hyperglycemia in diabetic KK-A(y) mice. Biochem Biophys Res Commun 344:597-604

32. Kahn BB, Alquier T, Carling D, Hardie DG (2005) AMP-activated protein kinase: ancient energy gauge provides clues to modern understanding of metabolism. Cell Metab 1:15-25

33. Ishizuka S, Tanaka S, Xu H, Hara H (2004) Fermentable dietary fiber potentiates the localization of immune cells in the rat large intestinal crypts. Exp Biol Med 229:876-884

34. Shimotoyodome A, Meguro S, Hase T, Tokimitsu I, Sakata T (2000) Short chain fatty acids but not lactate or succinate stimulate mucus release in the rat colon. Comp Biochem Physiol A Mol Integr Physiol 125:525-531
35. Everard A, Lazarevic V, Derrien M et al (2011) Responses of gut microbiota and glucose and lipid metabolism to prebiotics in genetic obese and diet-induced leptin-resistant mice. Diabetes 60:2775-2786

36. Ben-Shlomo S, Zvibel I, Shnell M et al (2011) Glucagon-like peptide-1 reduces hepatic lipogenesis via activation of AMPactivated protein kinase. J Hepatol 54:1214-1223

37. Kang K, Reilly SM, Karabacak V et al (2008) Adipocyte-derived Th2 cytokines and myeloid PPARdelta regulate macrophage polarization and insulin sensitivity. Cell Metab 7:485-495

38. Odegaard JI, Ricardo-Gonzalez RR, Red Eagle A et al (2008) Alternative M2 activation of Kupffer cells by PPARdelta ameliorates obesity-induced insulin resistance. Cell Metab 7:496-507

39. Spooner CJ, Sebastian T, Shuman JD et al (2007) C/EBPbeta serine 64, a phosphoacceptor site, has a critical role in LPSinduced IL-6 and MCP-1 transcription. Cytokine 37:119-127

40. Yamana J, Santos L, Morand E (2009) Enhanced induction of LPSinduced fibroblast MCP-1 by interferon-gamma: involvement of JNK and MAPK phosphatase-1. Cell Immunol 255:26-32

41. Jiang C, Ting AT, Seed B (1998) PPAR-gamma agonists inhibit production of monocyte inflammatory cytokines. Nature 391:8286

42. Ricote M, Li AC, Willson TM, Kelly CJ, Glass CK (1998) The peroxisome proliferator-activated receptor-gamma is a negative regulator of macrophage activation. Nature 391:79-82

43. Cancello R, Henegar C, Viguerie N et al (2005) Reduction of macrophage infiltration and chemoattractant gene expression changes in white adipose tissue of morbidly obese subjects after surgery-induced weight loss. Diabetes 54:2277-2286

44. Kováčiková M, Sengenes C, Kováčová Z et al (2011) Dietary intervention-induced weight loss decreases macrophage content in adipose tissue of obese women. Int $\mathrm{J}$ Obes 35:91-98 